\title{
Vortex Splitting in Subcritical Nonlinear Schrödinger Equations
}

\author{
Natalia G. Berloff * \\ Department of Applied Mathematics and Theoretical Physics \\ University of Cambridge, Cambridge, CB3 0WA, United Kingdom
}

\begin{abstract}
Vortices and axisymmetric vortex rings are considered in the framework of the subcritical nonlinear Schrödinger equations. The higher order nonlinearity present in such systems models many-body interactions in superfluid systems and allows one to study the effects of negative pressure on vortex dynamics. We find the critical pressure for which the straight-line vortex becomes unstable to radial expansion of the core. The energy of the straight-line vortices and energy, impulse and velocity of vortex rings are calculated. The effect of a varying pressure on the vortex core is studied. It is shown that under the action of the periodically varying pressure field a vortex ring may split into many vortex rings and the conditions for which this happens are elucidated. These processes are also relevant to experiments in BoseEinstein condensates where the strength and the sign of two-body interactions can be changed via Feshbach resonance.
\end{abstract}

Key words: superfluidity, vortices, vortex rings, nonlinear Schrödinger equation.

\section{Introduction}

The nonlinear Schrödinger (NLS) equation

$$
-i \psi_{t}=\frac{1}{2} \nabla^{2} \psi+g|\psi|^{2} \psi
$$

where $g= \pm 1$, is one of the most studied equations of mathematical physics with applications ranging from nonlinear optics, where $\psi$ represents the electric field, Bose-Einstein condensates (BECs) and superfluidity, where $\psi$ is the

* Email address: N.G.Berloff@damtp.cam.ac.uk 
field wavefunction, to fluid dynamics, where $\psi$ describes the amplitude of almost monochromatic wave. The nonlinear term in this equation represents the action of a refraction index that depends on the electric field intensity in nonlinear optics or corresponds to the two-body repulsive $(g=-1)$ or attractive interactions $(g=1)$ of the bosons in superfluids and BECs.

The formation of singularities in finite time for the focusing NLS equation (with $g=1$ ) is in practice been arrested by higher order nonlinear terms that come from the expansion of the nonlinear refraction index in optics, from many-body interactions in superfluids or from the effects of the geometry of the trapping potential in BECs that offers the reduction in the number of dimensions. These leads to a modification of the focusing NLS equation to what is known as "subcritical" or cubic-quintic NLS equation

$$
-\mathrm{i} \psi_{t}=\frac{1}{2} \nabla^{2} \psi+\left(g_{1}|\psi|^{2}-g_{2}|\psi|^{4}\right) \psi
$$

This model was used to study cavitation and vortex nucleation (Josserand et al, 1995), formation of vapor droplets in superfluids (Josserand and Rica, 1997), solitary waves in nonlinear optics (Kivshar and Agrawal, 2002), and bright solitons in elongated BECs (Sinha et al, 2006; Khaykovich and Malomed, 2006).

In this paper we consider a general class of subcritical NLS (SNLS) equations that allow for different orders of nonlinearity

$$
-\mathrm{i} \psi_{t}=\frac{1}{2} \nabla^{2} \psi+\left(g_{1}|\psi|^{2}-g_{2}|\psi|^{2(1+\gamma)}\right) \psi
$$

where $\gamma$ is an integer. The choice of the parameter $\gamma=1$ corresponds to the first-order term in the expansion of nonlinearity in the correlation energy and results in the the cubic-quintic equation (2), whereas $\gamma=3$ is close to the value 2.8 used in density-functional theories (Dalfovo, 1992) to produce a quantitatively correct equation of state for superfluid helium II.

Thus, we can view Eq. (3) to be a dimensionless form of a modified GrossPitaevskii equation

$$
\mathrm{i} \hbar \psi_{t}=-\frac{\hbar^{2}}{2 m} \nabla^{2} \psi+\left(W_{0}|\psi|^{2(1+\gamma)}-V_{0}|\psi|^{2}\right) \psi
$$

where $m$ is the mass of a boson, $V_{0}$ is a $\delta$-function two-body attractive interaction potential and many-body repulsive interactions are characterized by a parameter $W_{0}$. Equation 4 can be written in the Hamiltonian form

$$
\mathrm{i} \hbar \frac{\partial \psi}{\partial t}=\frac{\delta H}{\delta \psi^{*}}
$$


where the Hamiltonian (energy functional) of the system is

$$
H=\int_{V}\left(\frac{\hbar^{2}}{2 m}|\nabla \psi|^{2}-\frac{V_{0}}{2}|\psi|^{4}+\frac{W_{0}}{2+\gamma}|\psi|^{2(2+\gamma)}\right) d \mathbf{x} .
$$

To find the ground state the energy functional (6) has to be minimized subject to the conservation of the number of particles $N=\int|\psi|^{2} d \mathbf{x}$. This can be achieved by introducing a Lagrange multiplier $\mu$ and minimizing $H-\mu N$. The energy of the ground state becomes

$$
H_{0}=-\frac{V_{0}}{2} \frac{N^{2}}{V}+\frac{W_{0}}{2+\gamma} \frac{N^{2+\gamma}}{V^{1+\gamma}}
$$

and the ground state is given by $\psi_{0}^{2}=N / V, V=\int d \mathbf{x}$. The chemical potential $\mu$ can be introduced into (4) explicitly by

$$
\psi \rightarrow \psi \exp (-\mathrm{i} \mu t / \hbar)
$$

so that (4) becomes

$$
\mathrm{i} \hbar \psi_{t}=-\frac{\hbar^{2}}{2 m} \nabla^{2} \psi+\left(W_{0}|\psi|^{2(1+\gamma)}-V_{0}|\psi|^{2}-\mu\right) \psi
$$

The ground state, $\psi_{0}$, gives the value of the chemical potential as

$$
\mu=W_{0} \psi_{0}^{2(1+\gamma)}-V_{0} \psi_{0}^{2}
$$

The hydrostatic pressure of the system is found as

$$
P=-\frac{\partial H_{0}}{\partial V}=\frac{(1+\gamma) W_{0}}{2+\gamma} \psi_{0}^{2(2+\gamma)}-\frac{V_{0}}{2} \psi_{0}^{4}
$$

Hydrodynamic relation for the compressibility

$$
\frac{1}{m c^{2}}=\frac{\partial n}{\partial P}
$$

where $n=\left|\psi_{0}\right|^{2}$ is the number density, gives the expression for the speed of sound, $c$ as

$$
c^{2}=\frac{(1+\gamma) W_{0} n^{1+\gamma}-V_{0} n}{m}
$$

We conclude that the SNLS model, in particular, can be used to study the effects of a negative pressure. This is not possible with the cubic NLS model (1) that is often used as a phenomenological model of superfluid helium, but where $P \sim \rho^{2}$.

In the last couple of decades there has been a number of experiments which explore the behaviour of superfluid helium at negative pressure by means of 
ultrasound waves that produce an oscillating pressure within a small volume of helium (Nissen et al, 1989; Maris and Xiong, 1989; Xiong and Maris, 1989). In BECs experiments, Feshbach resonance is used to change the magnitude and sign of the scattering length. This can be modelled by Eq. (4) with a periodically varying $V_{0}(t)$ in the presence of the external magnetic trap. In this paper we shall study the effects of variations of $V_{0}$ on the vortex structure and dynamics.

We cast Eq. (9) into dimensionless form by

$$
\mathbf{x} \rightarrow b \mathbf{x}, \quad t \rightarrow \frac{\hbar}{W_{0} \psi_{0}^{2(1+\gamma)}} t, \quad \psi \rightarrow \psi_{0} \psi
$$

where the healing length, $b$, is defined by

$$
b=\frac{\hbar}{\sqrt{W_{0} m \psi_{0}^{2(1+\gamma)}}},
$$

so that Eq. (9) becomes

$$
-i \psi_{t}=\frac{1}{2} \nabla^{2} \psi+\left(|\psi|^{2(1+\gamma)}-2 \xi|\psi|^{2}+(1-2 \xi)\right) \psi
$$

where we denoted $\xi=V_{0} / 2 W_{0} \psi_{0}^{\gamma}$.

At $P=0, n_{0}=\left(V_{0}(2+\gamma) / 2(1+\gamma) W_{0}\right)^{1 / \gamma}$ and the speed of sound is $c_{0}^{2}=(1+$ $\gamma) \gamma W_{0} n_{0}^{1+\gamma} /(2+\gamma)$. The healing length becomes $b=\hbar / m c_{0} \sqrt{\gamma(1+\gamma) /(2+\gamma)}$. Since the known speed of sound in superfluid helium is approximately $238 \mathrm{~m}$ $\mathrm{s}^{-1}$, the healing length at zero pressure is $b=0.6685 \sqrt{\gamma(1+\gamma) /(2+\gamma)} \AA$, which for $\gamma=3$ gives $b=1 \AA$ and the unit of time as $6.28 \times 10^{-13} \mathrm{~s}$.

In dimensionless units, the wavefunction in the bulk is $\psi_{0}=1$, the density is $\rho=|\psi|^{2}$, and the local pressure and the local speed of sound are

$$
\begin{aligned}
P & =\frac{1+\gamma}{2+\gamma} \rho^{2+\gamma}-\xi \rho, \\
c & =\sqrt{(1+\gamma) \rho^{1+\gamma}-2 \xi \rho}
\end{aligned}
$$

The paper is organized as follows. In Section 2 we study the amplitude and energy of the straight-line vortex at various pressures (interactomic strengths). We find the critical value of $\xi$ at which the vortex becomes unstable to radial expansion for various values of $\gamma$. Section 3 is devoted to vortex rings and other travelling coherent structures that propagate with a fixed velocity. We calculate the energy and impulse of these matter waves. The time evolution of a line vortex and a vortex ring with a periodically varying pressure is studied 
in Section 4. We show that the vortex ring splits into many vortex rings if the negative pressure reaches the critical value for the vortex instability. We conclude with Section 5 .

\section{Vortex Lines}

A vortex line is defined by a zero of the wave function $\psi=0$. In cylindrical coordinates $(r, \theta, z)$ the wave function of the straight-line vortex takes form

$$
\psi=f(r) \exp [i s \theta]
$$

where $s$ is an integer ("winding number", "topological charge"). Fluid rotates around the $z$-axis with the tangential velocity

$$
\mathbf{u}=s \nabla \theta=\frac{s}{r} \mathbf{e}_{\theta}
$$

and the amplitude, $f$, satisfies

$$
\frac{1}{2}\left[\frac{1}{r} \frac{d}{d r}\left(r \frac{d f}{d r}\right)-\frac{s^{2}}{r^{2}} f\right]+2 \xi f^{3}+(1-2 \xi) f-f^{2(1+\gamma)+1}=0 .
$$

The boundary conditions are $f(0)=0$ and $f \rightarrow 1$ as $r \rightarrow \infty$. Analysis of Eq. (21) shows that near the origin $f(r) \sim a_{s} r^{|s|}$ and at infinity

$f(r) \approx 1-\frac{s^{2}}{4(1+\gamma-2 \xi) r^{2}}-\frac{s^{2}\left(2 \gamma^{2} s^{2}+\gamma\left(8+3 s^{2}\right)-\left(8+s^{2}\right)(2 \xi-1)\right)}{32(1+\gamma-2 \xi)^{3} r^{4}}+\cdots$

Similar to the case of the cubic defocusing NLS equation, only vortices with single unit of quantization, $s= \pm 1$, are dynamically stable (Josserand et al, 1995). Equation 21 was solved using a finite-difference discretization and Newton-Raphson iterations. Figure 1 gives plots of the vortex amplitudes $f$ as functions of $r$. Figure 2 depicts the slopes at the origin of the vortex amplitudes, $a_{1}$, as functions of $\xi$ for various values of $\gamma$. Above a critical value of $\xi_{\text {crit }}(\gamma)$ the vortex becomes unstable and expands in a radial direction as seen by numerically integrating Eq. (16) starting with the stable vortex and raising the value of $\xi$. The existence of a critical pressure for the vortex stability has been established in the context of density-functional theory by Xiong and Maris (1991) (giving the value of the critical pressure as -6.5 bars) and by a more sophisticated theory by Dalfovo (1992) (giving the value of -8 bars). We observe that the instability occurs when the slope of the vortex amplitude at the center of the vortex becomes zero and the negative pressure forces can no longer be balanced by the centrifugal energy of the fluid flow.

We can find the values of the critical pressure for the instability by evaluating 
Fig. 1. The amplitudes of straight-line vortices for $s= \pm 1$ as solutions of Eq. (21) for various $\gamma=1,2,3,4,5$ and $\xi=(1+\gamma) /(2+\gamma)$ that correspond to zero pressure. The larger values of $\gamma$ correspond to tighter vortex cores.

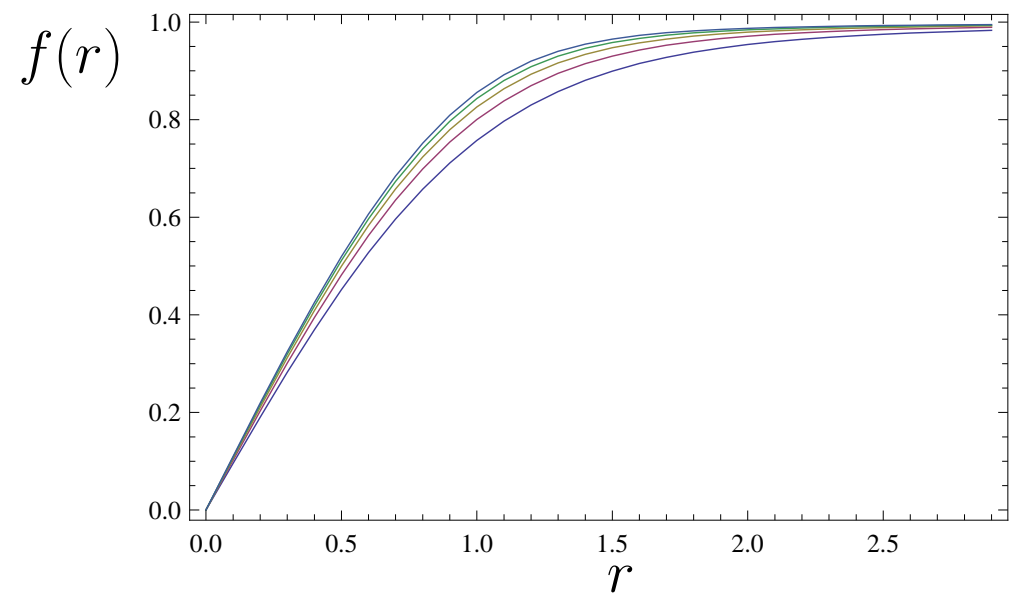

Fig. 2. The slopes at the origin, $a_{1}$, of the amplitudes of straight-line vortices for $s= \pm 1$ as solutions of Eq. (21) for various $\gamma=1,2,3,4$ as functions of $\xi$. Dots represent numerical integration, solid lines - Eq.(23). The values of $\left(\xi, a_{1}\right)$ that correspond to zero pressure are shown by red crosses.

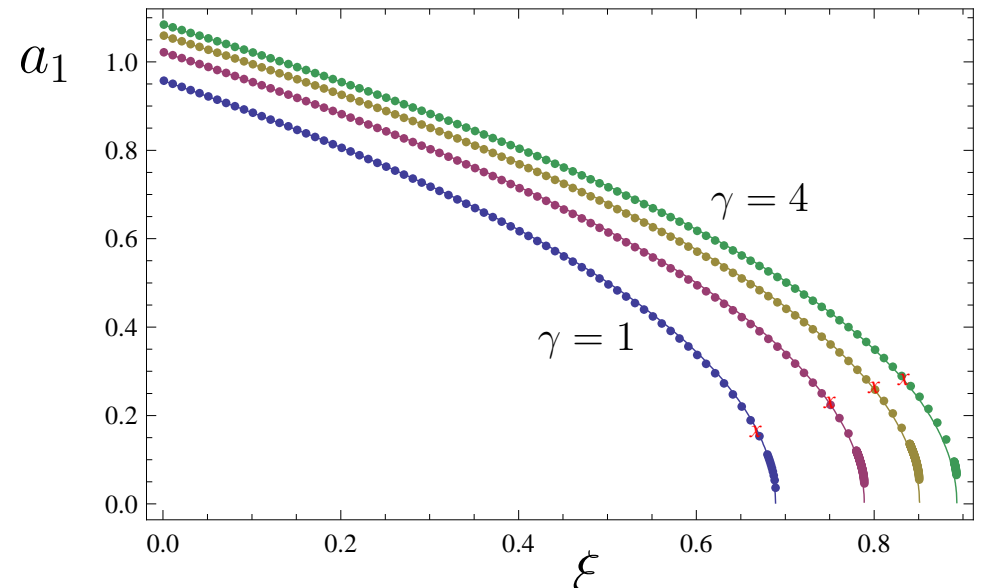

Eq. (17) with $\rho^{\gamma}=V_{0} / 2 W_{0} \xi_{\text {crit }}$ and calculating $W_{0}$ and $V_{0}$ at zero pressure using the speed of sound $238 \mathrm{~m} \mathrm{~s}^{-1}$, mass of the boson $6.628 \times 10^{-27} \mathrm{~kg}$ and the density $145.2 \mathrm{~kg} \mathrm{~m}^{-3}$. For $\gamma=3$ we obtained the critical pressure as -6 bar, that agrees quite well with the estimates using much more sophisticated density-functional theories.

Phenomenologically, the slope at the origin obeys a simple law

$$
a_{1}(\xi)=a_{1}(0) \sqrt{1-\xi / \xi_{\text {crit }}}
$$

where $a_{1}(0)=\lim _{\xi \rightarrow 0} a_{1}(\xi)$ and $\xi_{\text {crit }}=a_{1}^{-1}(0)$. Equation (23) gives the slope at the origin accurate to two significant digits. For instance, our calculations 
agree with the result presented by Josserand et al (1995) done for $\gamma=1$. Numerically, we get $a_{1}=0.286$ for $\xi=5 / 8$, and Eq. (23) gives $a_{1}=0.29$. Table 1 shows the values of $a_{1}(0)$ and $\xi_{\text {crit }}$ for various $\gamma$.

\section{Table 1.}

\begin{tabular}{|c|cccc|}
\hline & $\gamma=1$ & $\gamma=2$ & $\gamma=3$ & $\gamma=4$ \\
\hline$a_{1}(0)$ & 0.9575 & 1.02155 & 1.05921 & 1.08409 \\
$\xi_{\text {crit }}$ & 0.689 & 0.789 & 0.851 & 0.893 \\
\hline
\end{tabular}

To determine the energy of the vortex, we need to modify the energy functional (6) by the presence of the ground state. To do this we restore dimensional units temporarily. Following Jones and Roberts (1982) we denote by $\psi_{u}$ the wavefunction of the undisturbed system of the same mass, so that

$$
\int_{V}|\psi|^{2} d \mathbf{x}=\psi_{u}^{2} v,
$$

where $v=\int_{V} d \mathbf{x}$, and by $\psi_{\infty}$ the wavefunction of the bulk: $\psi \rightarrow \psi_{\infty}$ as $r \rightarrow \infty$. For the quartic term in the expression for the energy we write using Eq. (24)

$$
q_{2}=\int_{V}|\psi|^{4} d \mathbf{x}-\psi_{u}^{4} v=\int_{V}\left(|\psi|^{2}-\psi_{\infty}^{2}\right)^{2} d \mathbf{x}-\left(\psi_{u}^{2}-\psi_{\infty}^{2}\right)^{2} v
$$

Similarly, we obtained recursively

$$
\begin{gathered}
q_{3}=\int_{V}|\psi|^{6} d \mathbf{x}-\psi_{u}^{6} v=\int_{V}\left(|\psi|^{2}-\psi_{\infty}^{2}\right)^{3} d \mathbf{x}-\left(\psi_{u}^{2}-\psi_{\infty}^{2}\right)^{3} v+3 \psi_{\infty}^{2} q_{2} \\
q_{2+n}=\int_{V}|\psi|^{2(2+n)} d \mathbf{x}-\psi_{u}^{2(2+n)} v=\int_{V}\left(|\psi|^{2}-\psi_{\infty}^{2}\right)^{2+n} d \mathbf{x} \\
-\left(\psi_{u}^{2}-\psi_{\infty}^{2}\right)^{2+n} v+\sum_{k=1}^{n}(-1)^{k-1} C_{2+n}^{k} \psi_{\infty}^{2 k} q_{2+n-k}
\end{gathered}
$$

where $n$ is a positive integer and $C_{i}^{k}$ are the binomial coefficients. The terms $\left(\psi_{\infty}^{2}-\psi_{u}^{2}\right)^{n} v$ are $O(1 / v)$ and vanish as $v \rightarrow \infty$. In this limit, the dimensionless energy becomes

$$
E_{\gamma}=\frac{1}{2} \int|\nabla \psi|^{2} d \mathbf{x}-\xi \int\left(\left|\psi^{2}\right|-1\right)^{2} d \mathbf{x}+\frac{1}{2+\gamma} Q_{2+\gamma}
$$

where $Q_{2+\gamma}$ are defined recursively as

$$
\begin{aligned}
Q_{2} & =\int\left(|\psi|^{2}-1\right)^{3} d \mathbf{x} \\
Q_{2+n} & =\int\left(|\psi|^{2}-1\right)^{2+n} d \mathbf{x}+\sum_{k=1}^{n}(-1)^{k-1} C_{2+n}^{k} Q_{2+n-k}
\end{aligned}
$$


Fig. 3. Values of vortex core parameter $\ell$ defined by (31) of straight-line vortices for $s= \pm 1$ for various $\gamma=1,2,3,4$ as functions of $\xi$. The values of the vortex core parameter $\ell$ at zero pressure are -0.09 for $\gamma=1,0.22$ for $\gamma=2,0.37$ for $\gamma=3$, 0.46 for $\gamma=4$. The phenomenological fit (32) is given by the solid lines.

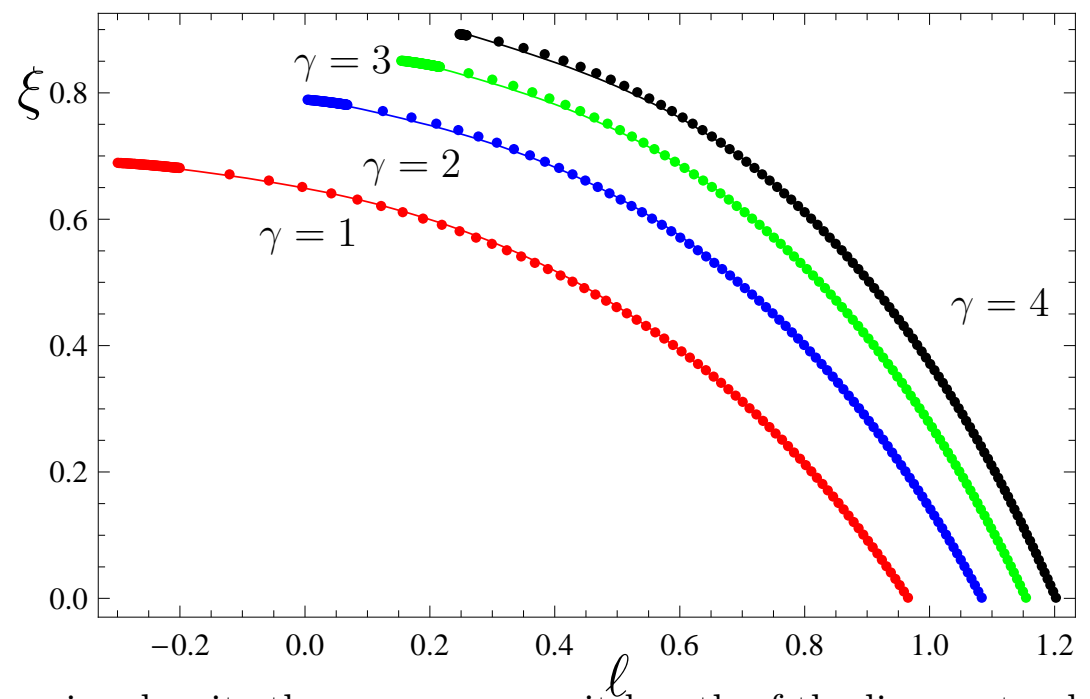

In dimensional units the energy per unit length of the line vortex becomes

$$
\begin{aligned}
E_{v} & =\frac{\kappa^{2} \rho_{\infty}}{4 \pi}\left(\int_{0}^{\infty}\left[\left(\frac{d R}{d r}\right)^{2}+\frac{R^{2}}{r^{2}}\right] r d r-2 \xi \int_{0}^{\infty}\left(R^{2}-1\right)^{2} r d r\right. \\
& +\frac{2}{2+\gamma}\left[\int_{0}^{\infty}\left(R^{2}-1\right)^{2+\gamma} r d r\right. \\
& \left.\left.+\sum_{k=1}^{\gamma}(-1)^{k-1} C_{2+\gamma}^{k} \int_{0}^{\infty}\left(R^{2}-1\right)^{2+\gamma-k} r d r\right]\right)
\end{aligned}
$$

The second term in the first integral in Eq. (29) represents the classical kinetic energy that diverges. This can be remedied by introducing a cut-off distance $L$, corresponding to the characteristic size of the container, and writing

$$
\int_{0}^{\infty} \frac{R^{2}}{r} d r=\int_{1}^{L / b} \frac{1}{r} d r+\int_{0}^{1} \frac{R^{2}}{r} d r+\int_{1}^{\infty} \frac{R^{2}-1}{r} d r
$$

The energy per unit length of the line vortex can, therefore, be expressed in the form

$$
E_{v}=\frac{\kappa^{2} \rho_{\infty}}{4 \pi}\left[\ln \left(\frac{L}{b}\right)+\ell\right]
$$

where $\ell$ can be found by numerical integration. Figure 3 shows the values of the vortex core parameter $\ell$ as a function of $\xi$ for various values of $\gamma$.

Phenomenologically, the relationship between $\xi$ and $\ell$ has the form

$$
\xi=\xi_{\text {crit }}-\sigma_{1}\left(\ell-\ell_{\text {crit }}\right)-\sigma_{2}\left(\ell-\ell_{\text {crit }}\right)^{3}
$$


where $\ell_{\text {crit }}$ is the value of $\ell$ at $\xi_{\text {crit }}$. The values of parameters $\sigma_{i}$ and $\ell_{\text {crit }}$ for various $\gamma$ are given in Table 2 .

\section{Table 2.}

The coefficients of Eq.(32) that define the vortex core parameter $\ell$.

\begin{tabular}{|c|cccc|}
\hline & $\gamma=1$ & $\gamma=2$ & $\gamma=3$ & $\gamma=4$ \\
\hline$\ell_{\text {crit }}$ & -0.299578 & 0.00410 & 0.15429 & 0.247549 \\
$\sigma_{1}$ & 0.117906 & 0.20718 & 0.261971 & 0.298006 \\
$\sigma_{2}$ & 0.264568 & 0.449719 & 0.590682 & 0.701111 \\
\hline
\end{tabular}

\section{Vortex Rings}

In this section we consider the circular vortex rings that propagate in $z$-direction preserving their form. For large vortex rings with the radius $R$ much greater than the size of the core characterized by the healing length $b$ the energy can be found by using the energy of the straight line vortex (31) in the region close to the vortex line and using only the kinetic energy term in the region away from the vortex line. The sum of these two contributions to energy gives a simple result (Amit and Gross, 1966; Roberts and Grant, 1971)

$$
E=\frac{1}{2} \kappa^{2} \rho_{\infty} R\left[\ln \left(\frac{8 R}{b}\right)-2+\ell\right]
$$

The momentum of the large vortex ring becomes

$$
\mathbf{p}=\kappa \rho_{\infty} \pi R^{2} \mathbf{e}_{\mathbf{z}}
$$

where $\mathbf{e}_{\mathbf{z}}$ is the unit vector in the direction of the ring motion.

The vortex rings and other travelling wave structures that propagate with a constant velocity correspond to stationary solutions of the SNLS equations in the frame of reference moving with the velocity of the ring. The wavefunction satisfies

$$
\mathrm{i} U \frac{\partial \psi}{\partial z}=\frac{1}{2} \nabla^{2} \psi+\left(|\psi|^{2(1+\gamma)}-2 \xi|\psi|^{2}+(1-2 \xi)\right) \psi
$$

We can perform a variation $\psi \rightarrow \psi+\delta \psi$ in the expressions for momentum (see Jones and Roberts, 1982)

$$
\mathbf{p}=\frac{1}{2 \mathrm{i}} \int\left[\left(\psi^{*}-1\right) \nabla \psi-(\psi-1) \nabla \psi^{*}\right] d \mathbf{x}
$$


and energy (27) and using (35) show that $\delta E=U \delta p$, or

$$
U=\frac{\partial E}{\partial p}
$$

We can differentiate Eqs. (33) and (34) with respect to $R$ and after substitution into Eq. (37) obtain the expression for the velocity of the large vortex ring as

$$
U=\frac{\kappa}{4 \pi R}\left[\ln \left(\frac{8 R}{b}\right)-1+\ell\right]
$$

We can also use the Hamiltonian group relation (37) to derive an alternative form of the energy functional (27). We substitute $z \rightarrow \alpha z$, for a constant $\alpha$ in Eqs (27) and (36). Then using the variational relationship

$$
\left.\frac{\partial}{\partial \alpha} \delta(E-U p)\right|_{\alpha=1}=0
$$

gives

$$
E=\int\left|\frac{\partial \psi}{\partial z}\right|^{2} d \mathbf{x}
$$

As the radius of the vortex ring decreases the expressions (33), (34) and (38) are no longer accurately describe the energy, impulse and velocity of the ring. The sequence of the small vortex rings and other localized disturbances can be found numerically by a Newton-Raphson iteration technique. The infinite domain is mapped by the transformations $\widehat{x}=\tan ^{-1}(D x)$ and $\widehat{y}=\tan ^{-1}(D y)$ to a finite grid $\left(-\frac{\pi}{2}, \frac{\pi}{2}\right) \times\left(-\frac{\pi}{2}, \frac{\pi}{2}\right)$. $D$ is a constant chosen to lie in the range $D \sim$ 0.4-0.8. The resulting equations are expressed in second-order finite-difference form. Taking $201^{2}$ grid points in the finite domain, the resulting non-linear equations are solved by a Newton-Raphson iteration procedure using a banded matrix linear solver based on the bi-conjugate gradient stabilized iterative method. The accuracy of the obtained solutions is verified by evaluating the integral identities (37) and (40).

The families of the travelling wave solutions found are qualitatively similar to the sequence of such waves found by Jones and Roberts (1982) for the GrossPitaevskii model. They calculated the energy $E$ and momentum $p$ and showed that the sequence in the $p-E$ plane is given by two branches meeting at a cusp where $p$ and $E$ assume their minimum values, $p_{m}$ and $E_{m}$. As $p \rightarrow \infty$ on each branch, $E \rightarrow \infty$. On the lower branch the solutions were asymptotic to the large vortex rings. As $E$ and $p$ decrease from infinity along the lower branch, the solutions begin to lose their similarity to large vortex rings, and Eqs. (33), (34) and (38) determine $E, p$, and $U$ less and less accurately. Eventually, for a momentum $p_{0}$ slightly greater than $p_{m}$, the rings lose their vorticity $(\psi$ loses its zero), and thereafter the solitary solutions may better be described as 'rarefaction waves'. The upper branch consists entirely of these and, as 
Fig. 4. Families of travelling wave solutions of the subcritical NLS equation (16) for $\gamma=1$ (red thin solid line), $\gamma=2$ (blue thick solid line), and $\gamma=3$ (black dashed line) and (a) $\xi=1 / 2$ and (b) $\xi=(1+\gamma) /(2+\gamma)$. Dots show the positions of the transition between a vortex ring and a rarefaction pulse on the lower branches. The critical velocities at which the vortex ring becomes the rarefaction pulse are given in Table 3.
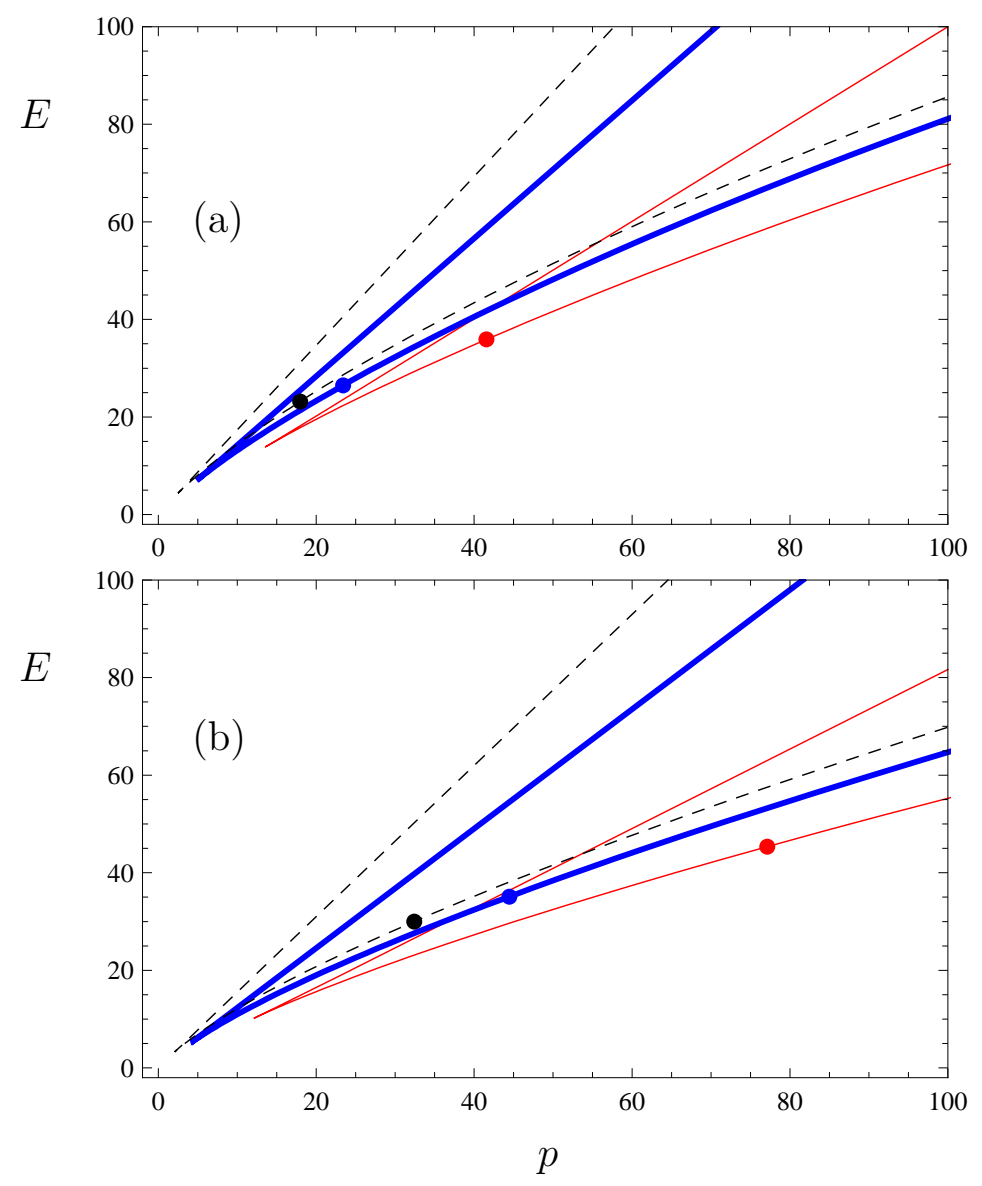

$p \rightarrow \infty$ on this branch, the solutions asymptotically approach infinitesimal sound waves. Figure 4 shows the positions of such cusps for the SNLS equations (16) for two values of $\xi: \xi=1 / 2$ and $\xi=(1+\gamma) /(2+\gamma)$. The critical velocities at which the vortex ring becomes the rarefaction pulse are given in Table 3 .

In Berloff and Roberts (1999) we discussed how a nonlocal NLS equation with a higher order nonlinearity brings the vortex core parameter $\ell$ and the healing length $b$ into the agreement. We observe that the same harmony is achieved by the subcritical NLS model (16) for $\gamma=3$. At zero pressure that gives $b=1$ $\AA$, the same value as the one found from the sound speed in Section 1. This also agrees with the experiments of Rayfield and Reif (1964), where the vortex ring travelling at $27 \mathrm{~cm} \mathrm{~s}^{-1}$ has the energy $10 \mathrm{eV}$.

\section{Table 3.}


The critical velocity of the transition between a vortex ring and a rarefaction pulse.

\begin{tabular}{|c|ccc|}
\hline$\xi$ & $\gamma=1$ & $\gamma=2$ & $\gamma=3$ \\
\hline$\frac{1}{2}$ & 0.7 & 0.91 & 1.03 \\
$\frac{1+\gamma}{2+\gamma}$ & 0.45 & 0.6 & 0.7 \\
\hline
\end{tabular}

\section{Vortex splitting}

In this section we discuss a novel mechanism of creating vorticity in superfluids. Previously, the semi-classical formation of vortices has been attributed to one of the four basic mechanisms: due to the existence of critical velocities (Frisch et al, 1992), due to the transverse instabilities of dispersive waves (Kuznetsov and Rasmussen, 1995), due to collapse of cavities (Berloff and Barenghi, 2004), due to energy transfer between waves (Berloff, 2004) and during the condensation (Berloff and Svistunov, 2002). Here we show that there exists another mechanism of vortex formation based on the expansion and collapse of the vortex core.

A negative pressure is generated in superfluid helium II by an ultrasonic transducer that is used to produce periodic sound pulses (Maris, 1994). This technique has been extremely successful in detecting electron bubbles in superfluids (Ghosh and Maris, 2005). It has been shown that the tensile strength of superfluid helium is much less than predicted by theory and this suggests that the quantized vortices may play a role in the cavitation process.

To consider the dynamics of a straight line vortex and a vortex ring in superfluid under the time-varying pressure field, we integrated Eq. (16) numerically forward in time. We used the 4 th order accurate finite difference scheme in space and the 4 th order Runge-Kutta in time.

We start by considering a single straight line vortex under the action of a varying pressure field controlled by parameter $\xi$. The pressure oscillates according to Eq. (17) with

$$
\xi(t)=\xi_{0}+\epsilon \sin (\pi t / 2 \eta)
$$

where $\epsilon$ is a small parameter and $\xi_{0}$ corresponds to the initial zero pressure $\xi_{0}=(1+\gamma) /(2+\gamma)$. If $\xi_{0}+\epsilon \leq \xi_{\text {crit }}$, then the vortex never becomes unstable and the size of the core increases until $t=\eta$ and decreases back to the unperturbed value at $t=2 \eta$ in response to the applied pressure. If $\xi_{0}+\epsilon>\xi_{\text {crit }}$, then the vortex core continues to increase while pressure is negative. This instability becomes arrested by the growing positive pressure, so the vortex core returns 
Fig. 5. Density contour plots of the vortex under the action of periodically varying pressure field characterized by Eq. (41) with $\epsilon=1 / 20, \gamma=1$ and $\eta=100$. The centre of the vortex is seen as black region, intermediate densities are shown in light gray, larger densities in dark gray.
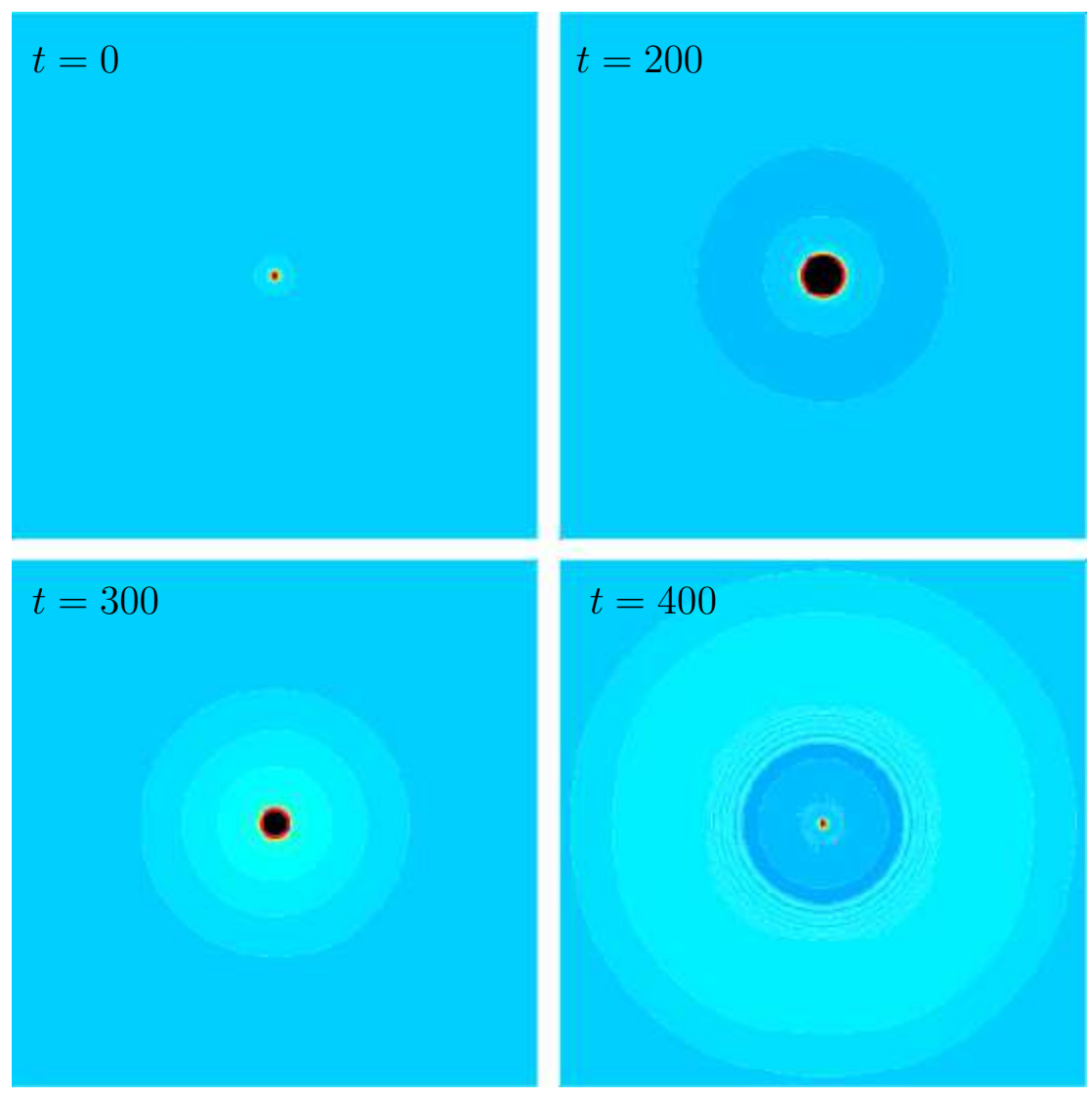

to the unperturbed value at $t=4 \eta$. Figure 5 shows the density contour plots for the dynamics of the vortex for $\epsilon=1 / 20, \gamma=1$ and $\eta=100$. The field around the vortex remains radially symmetric and there is no vortex splitting. The extra energy gained is emitted by the vortex core radially as sound waves.

To understand the action of the pressure on the vortex we calculated the energy of the system which in our dimensionless units is (see Eq. (31))

$$
E=\pi[\ln (L / b)+\ell(t)] .
$$

Figure 6 shows the plots of $\ell(t)$ as a function of $t$. The corresponding values of pressure are given by dashed line (and magnified by a factor of 100 for $\eta=100$ and by a factor of 500 for $\eta=200$.). The energy of the system, characterized by $\ell(t)$, increases between pressure minimum and maximum. To follow the evolution of the vortex core we plot the density of the field in the radial direction on Fig. 7 for $\epsilon=1 / 20, \gamma=1$ and $\eta=100$. The vortex core grows while the pressure stays negative (until $t=2 \eta$ ), after that the core 
Fig. 6. The vortex core parameter $\ell(t)$ in (42) as a function of time for the dynamics of the vortex under the action of periodically varying pressure field characterized by Eq. (41) with $\epsilon=1 / 20$ and $\gamma=1$ (solid lines). Dashed line gives the evolution of pressure given by Eq.(17) magnified by a factor of 100 (top panel) or 500 (bottom panel).
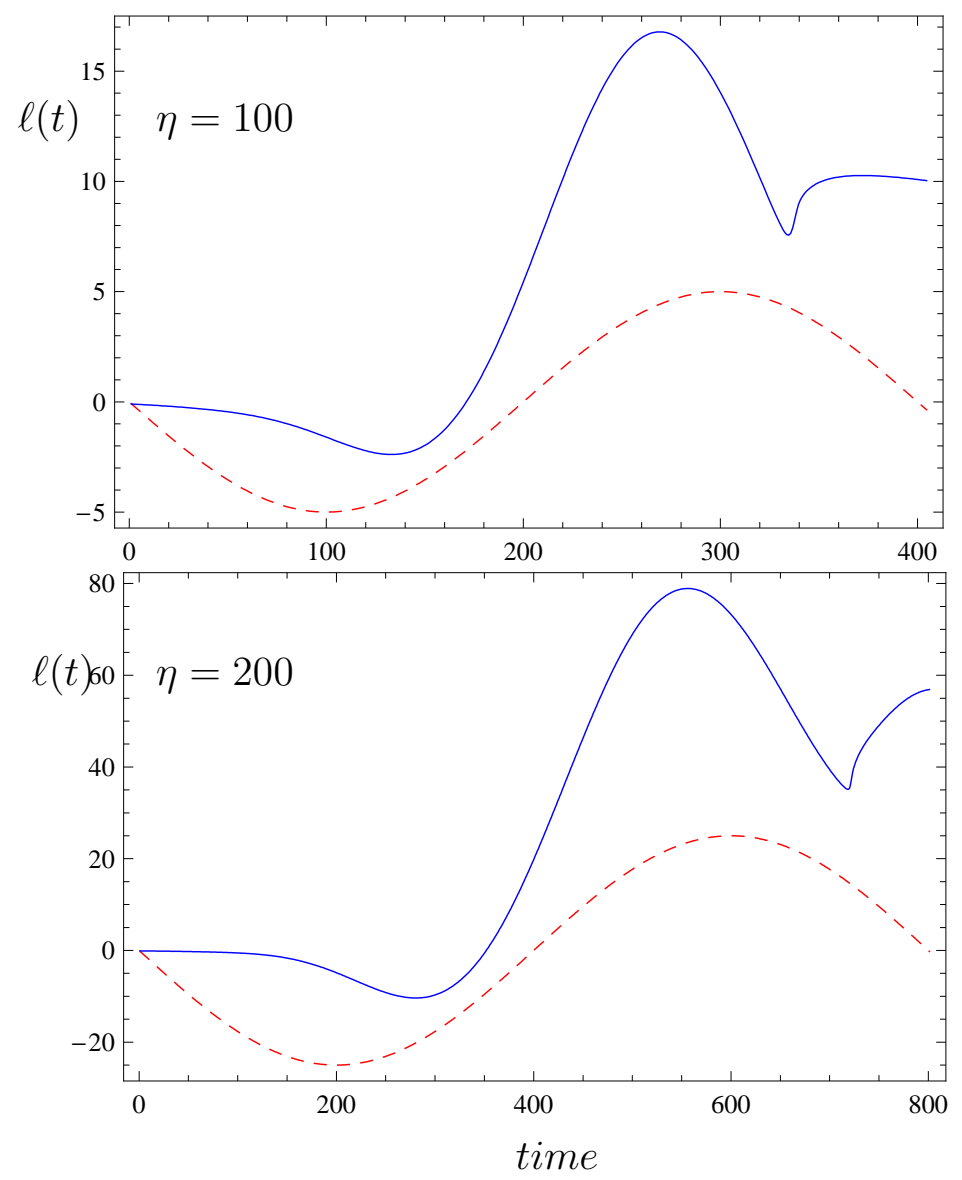

stabilizes and then decreases in size as pressure increases; see the top panel of Fig. 7. For positive pressures the core continues to decrease in size while having an energy density much larger than the energy of the stationary vortex at a given positive pressure; a large density accumulates at the vortex core at $t=340$; see the bottom panel of Fig. 7 . This extra energy is emitted by the vortex core as outgoing sound wave packet as it tries to regain its stationary density profile; see $t=350$ density profile at the bottom panel of Fig. 7 . The increase in the period of the pressure oscillations leads to qualitatively similar scenario. The difference is that the energy of the vortex has a longer growth period and, therefore, reaches larger values; see the bottom panel of Fig. 6.

For the vortices of large radii the effect of the pressure varying field is similar to that on the straight-line vortex, but the velocity field created by the vortex loop changes the pressure distribution around the core. The vortex core collapses faster where the velocity is lower leading to the vortex splitting as Figs. 
Fig. 7. Time snapshots of the density of a straight-line vortex under the action of varying pressure field characterised by Eq. (41) with $\epsilon=1 / 20$. On the top panel the times are $0,50,100,150,200,250,300$; on the bottom panel the times are $300,310,320,330,340,350$. The thinner lines correspond to later times.
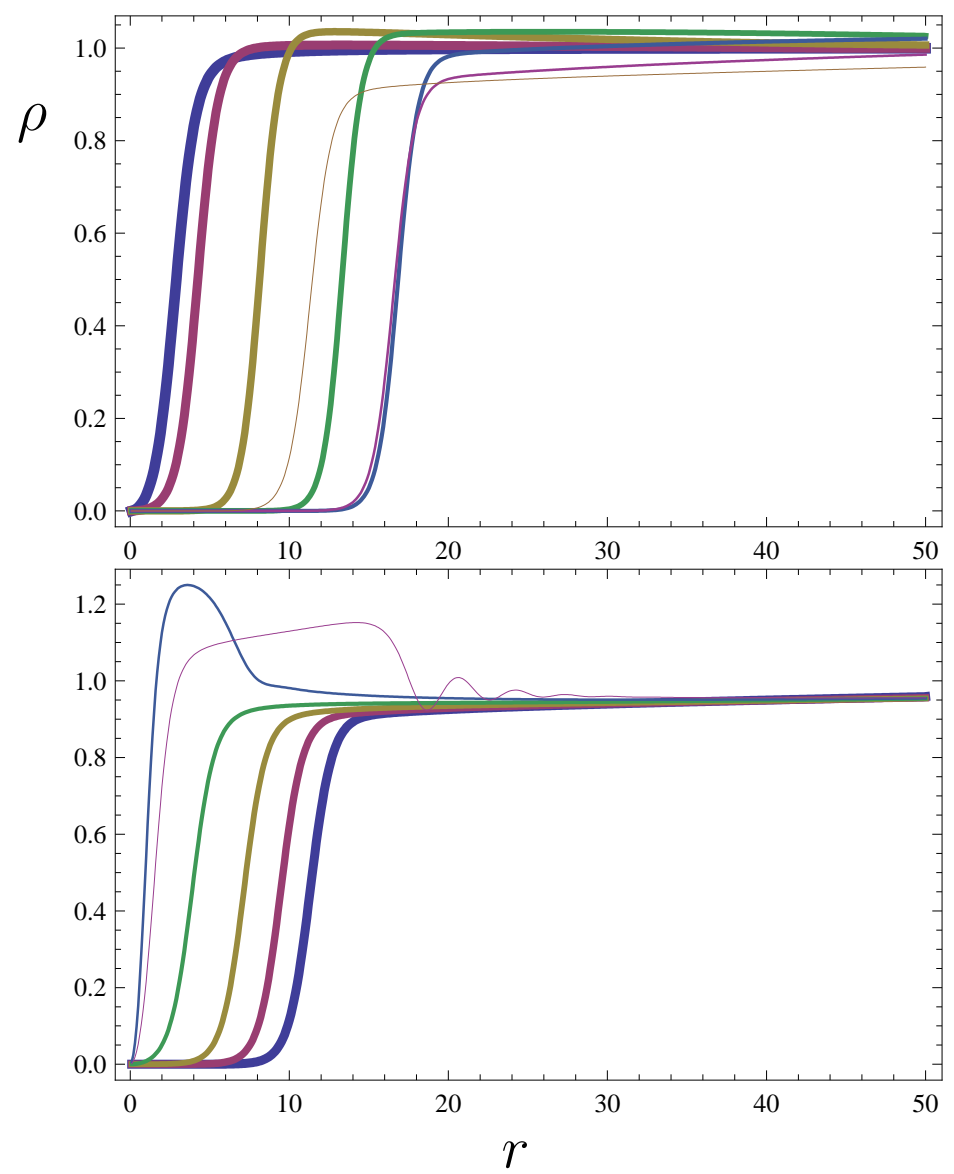

8 and 9 illustrate for $\gamma=1$ and $\gamma=3$ respectively. In both cases we start with a vortex ring of radius 50 at zero pressure. The parameter $\xi$ varies according to Eq. (41) with $\epsilon=1 / 20$ (for $\gamma=1$ ), $\epsilon=1 / 10$ (for $\gamma=3$ ) and $\eta=100$. The vortex ring splits into three rings (two of positive circulation and one ring with the negative circulation) on Fig. 8. There are many more vortex rings generated on Fig. 9. We can roughly estimate the number of vortex rings that will be created by calculating the available energy just before splitting. Under the action of the pressure field characterized by (41) with $\epsilon=1 / 20, \gamma=1$ and $\eta=100$, the single straight-line vortex emits sound when $\ell \approx 8$ (seen as a sharp cusp on Fig. (6)). The total energy available can be estimated from Eq. (27) and for $R=50$ is about 3 times the energy of one vortex ring of radius 50 at zero pressure. 
Fig. 8. Density contour plots of the cross section of the vortex ring under the action of periodically varying pressure field characterized by (41) with $\epsilon=1 / 20, \eta=100$ and $\gamma=1$. The cross-sections of vortex rings are seen as black dots, intermediate densities are shown in light gray, larger densities in dark gray. An initial vortex of radius $50 b$ is split into three vortex rings at the end of one period of pressure oscillations.
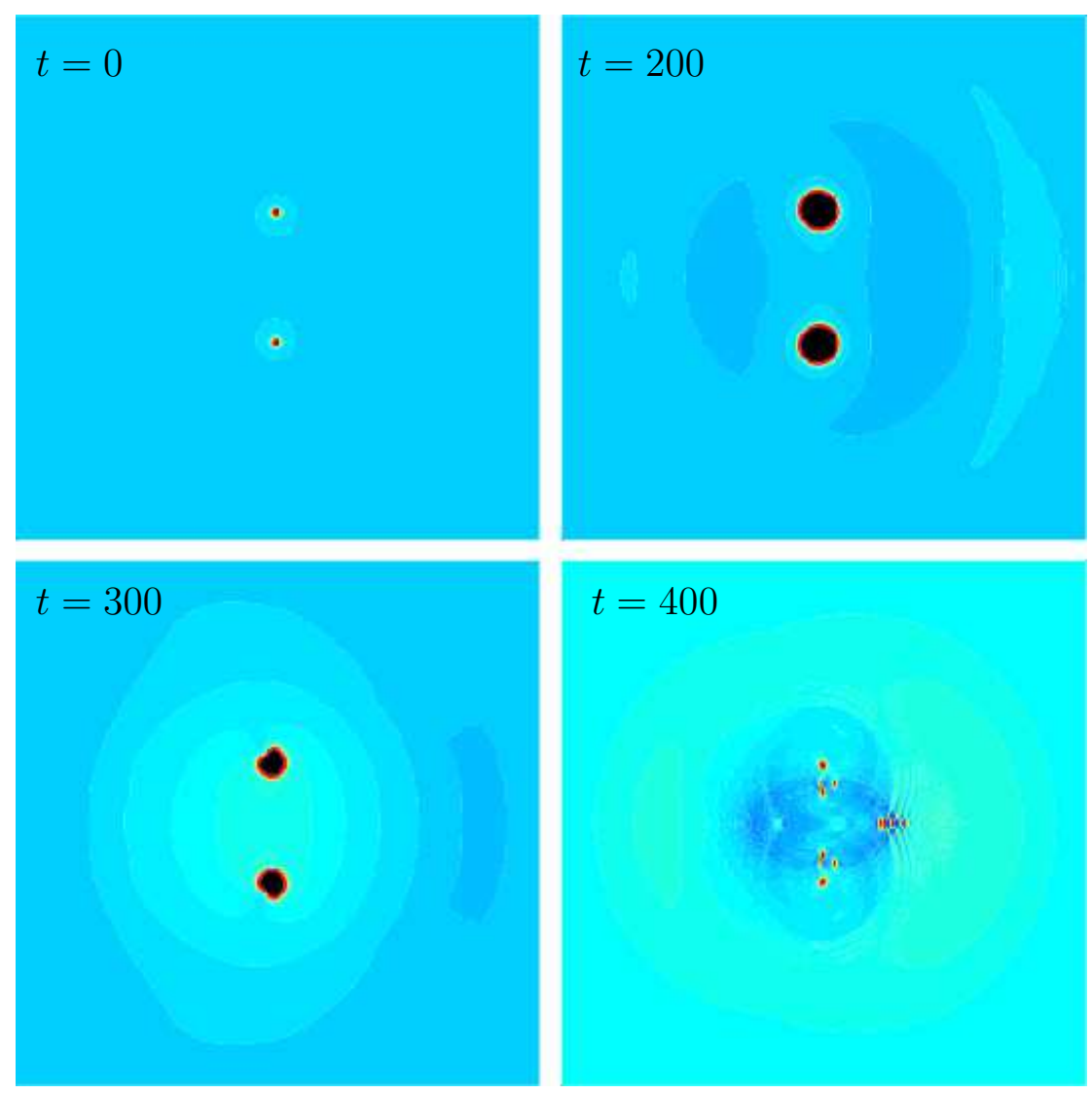

\section{Conclusions}

In summary, we considered the families of straight-line vortices and axisymmetric vortex rings in the framework of subcritical NLS equations with a focusing cubic nonlinearity and defocusing higher order term, characterized by the parameter $\gamma$; see Eq. (16). These models can be used to study the effects of negative pressure in superfluids and capture the dynamics of the many body systems with attractive two-body interactions such as spin-polarized $\mathrm{Li}_{7}$ (Bradley et al, 1995; Sackett et al, 1998). The strength of the two-body interactions as well as the hydrodynamic pressure are characterized by the parameter $\xi$.

We determined the structure and energy of the straight-line vortices and showed that the vortex becomes unstable to uniform radial expansion when the slope of the amplitude at the vortex center becomes zero and calculated 
Fig. 9. Density contour plots of the cross section of the vortex ring under the action of periodically varying pressure field characterized by Eq.(41) with $\epsilon=1 / 10, \gamma=3$ and $\eta=100$. The cross-sections of vortex rings are seen as black dots, intermediate densities are shown in light gray, larger densities in dark gray. An initial vortex of radius $50 b$ is split into many vortex rings at the end of one period of pressure oscillations.
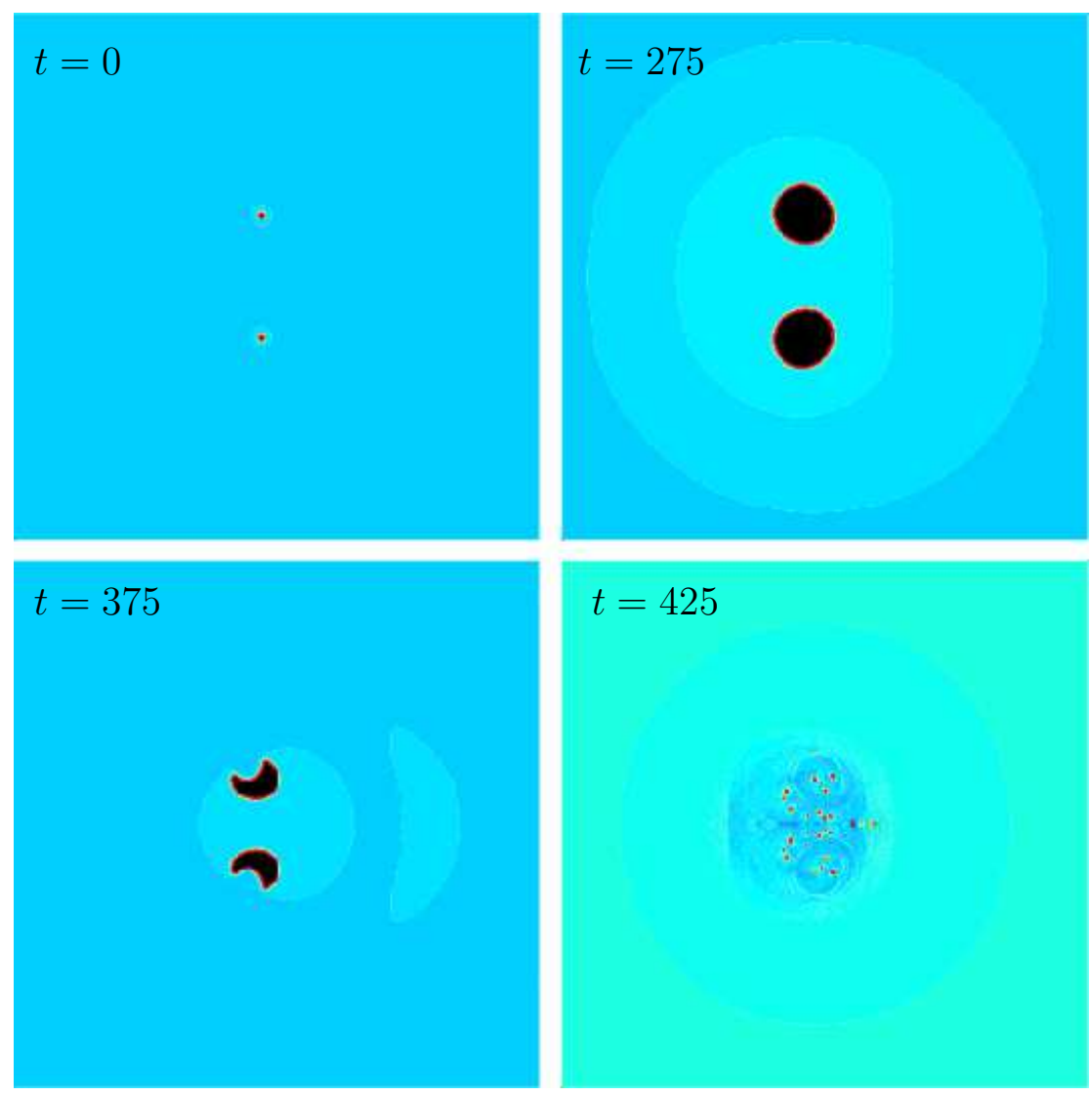

the critical value of $\xi$ for this instability. The corresponding critical pressure for $\gamma=3$ is close to the one obtained by much more sophisticated densityfunctional theories. We calculated the energy, impulse and velocity of the axisymmetric vortex rings and showed the families of the travelling coherent structures on the impulse-energy plane.

We considered the periodic oscillations of the parameter $\xi$ in time and elucidated its effect on the vortex core. There are three possible scenarios of the dynamics of the vortex.

1. If the value of $\xi$ never reaches the critical value for the vortex instability, then the vortex core simply adjusts to the varying pressure. As pressure decreases (increases), the vortex size grows (shrinks).

2. If the value of $\xi$ exceeds the critical value during the time evolution, then the vortex core grows while pressure stays negative and continues to do so until shortly before pressure reaches its maximum positive value. After that 
the vortex core shrinks and the extra energy is emitted as outgoing sound waves.

3. Same as in item 2 , but in the presence of large variations of the velocity field around the core, such as in the case of the vortex rings, the vortex core breaks into odd number of vortices (vortex rings) to preserve the total unit of circulation of \pm 1 around the initial vortex.

Finally, we suggest that the processes of the vortex instability and vortex splitting can be seen in trapped BECs where the magnitude and sign of the scattering length, which is represented by $\xi$ in our model, are changed by Feshbach resonance. In particular, our results indicate that there is a critical value of the interatomic attractive interactions for which a straight-line vortex becomes unstable. It seems likely that this instability can be arrested by the presence of a harmonic trapping potential. By periodically varying the sign and the magnitude of the two-body interactions in the trapped BEC it may be possible to produce the vortex spitting if the trap is not too close to being perfectly axisymmetric.

\section{Acknowledgments}

The author gratefully acknowledges the financial support from the EPSRCUK and useful discussions with Humphrey Maris.

\section{References}

Amit D. and Gross E. P. 1966. Vortex Rings in a Bose Fluid, Phys. Rev. 145, 130.

Berloff N.G and Roberts P. H. 1999. Motions in a Bose condensate VI. Vortices in a nonlocal model, J. of Phys. A: Math. and Gen., 32, 1-15.

Berloff N.G. and Barenghi C.F., 2004. Vortex nucleation by collapsing bubbles in Bose-Einstein condensates, Phys. Rev. Lett., 93, 090401.

Berloff N.G. 2004. Pade approximations of solitary wave solutions of the GrossPitaevskii equation, J. of Phys. A: Math. and Gen., 37, 1617 - 1632.

Berloff N.G. and Svistunov B. V. 2002. Scenario of strongly non-equilibrated Bose-Einstein condensation, Phys. Rev. A, 66, 013603.

Bradley C.C., Sackett C. A., Tollett J. J., and Hulet R. G. 1995. Evidence of Bose-Einstein Condensation in an Atomic Gas with Attractive Interactions, Phys. Rev. Lett. 75, 1687.

Dalfovo F, 1992. Structure of vortices in helium at zero temperature, Phys. Rev. B 46, 5482 - 5488 . 
Frisch T, Pomeau Y, and Rica S 1992. Transition to dissipation in a model of superflow, Phys. Rev. Letts. 69 1644-1648.

Jones C A and Roberts P H 1982. Motions in a Bose condensate: IV. Axisymmetric solitary waves, J. Phys. A: Gen. Phys. 152599 -2619.

Josserand C., Pomeau Y., and Rica S., 1995. Cavitation versus Vortex Nucleation in a Superfluid Model. Phys. Rev. Lett. 75, 3150.

Josserand C. and Rica S. 1997. Coalescence and Droplets in the Subcritical Nonlinear Schrdinger Equation, Phys. Rev. Lett. 78, 1215.

Ghosh A. and Maris H. J. 2005. Observation of a New Type of Electron Bubble in Superfluid Helium, Phys. Rev. Lett. 95, 265301.

Khaykovich L. and Malomed B. A., 2006. Deviation from one dimensionality in stationary properties and collisional dynamics of matter-wave solitons, Phys. Rev. A 74, 023607.

Kivshar Yu. S. and Agrawal G., Optical Solitons: From Fibers to Photonic Crystals (Academic Press, San Diego, 2003).

Kuznetsov E.A. and Juul RasmussenJ. 1995. Instability of two-dimensional solitons and vortices in defocusing media, Phys. Rev. E 51, 4479.

Maris H. J. and Xiong Q. 1989. Nucleation of bubbles in liquid helium at negative pressure, Phys. Rev. Lett. 63, 1078;

Maris H.J. 1994. Nucleation of Bubbles on Quantized Vortices in Helium-4, J. Low Temp. Phys. 94, 125-144.

Nissen J. A., Bodegom E. and Semura J. S., 1989. Tensile strength of liquid 4He, Phys. Rev. B 40, 6617.

Rayfield G. W and Reif F. 1964. Quantized Vortex Rings in Superfluid Helium, Phys. Rev. 136, A1194-1208.

Roberts P. H. and Grant J. 1971, Motions in a Bose condensate. I. The structure of the large circular vortex, J. Phys. A: Gen. Phys. - bf 4, 55-72.

Sackett A., Stoof H.T., and Hulet R.G.1998. Growth and Collapse of a BoseEinstein Condensate with Attractive Interactions, Phys. Rev. Lett. 80, 2031.

Sinha S., Cherny A. Yu., Kovrizhin D., and Brand J. 2006. Friction and Diffusion of Matter-Wave Bright Solitons, Phys. Rev. Lett. 96, 030406.

Xiong Q. and Maris H. J. 1989. Liquid helium at negative pressures: Nucleation of bubbles and anomalous phonon dispersion, J. Low Temp. Phys. 77, 347; 82, 105 (1991) 